EUROPEAN JOURNAL OF PURE AND APPLIED MATHEMATICS

Vol. 11, No. 1, 2018, 244-259

ISSN 1307-5543 - www.ejpam.com

Published by New York Business Global

\title{
Weak PS-rings over Skew Hurwitz Series
}

\author{
Mohamed A. Farahat ${ }^{1,2, *}$, Salha T. Al-Bogamy ${ }^{1}$ \\ 1 Department of Mathematics and Statistics, Faculty of Science, Taif University, Taif, \\ El-Haweiah, Kingdom of Saudi Arabia \\ 2 Mathematics Department, Faculty of Science, Al-Azhar University, Cairo, Egypt
}

\begin{abstract}
The notion of PS-rings is extended to the class of weak PS-rings. We explore the algebraic properties of such class and study its relation with some other rings such as a local ring and a semisimple NI-ring. Also, we show the following result concerning, the ring of skew Hurwitz series, $A=(H R, \sigma)$ : Let $R$ be a $\sigma$-compatible NI-ring with nil $(R)$ nilpotent, $\sigma(e)=e$ for every idempotent $e \in R$ and $R$ a torsion free as a $\mathbb{Z}$-module. If $R$ is a weak right PS-ring, then $A=(H R, \sigma)$ is a weak right PS-ring.
\end{abstract}

2010 Mathematics Subject Classifications: 06F05, 16W60, 16D25, 16P60

Key Words and Phrases: PS-modules, PS-rings, skew Hurwitz series rings

\section{Introduction}

All rings are assumed to be an associative ring with identity and modules are nonzero unitary right modules, unless otherwise stated. For a nonempty subset $X$ of $R, \mathrm{r}_{R}(X)$ (or $\ell_{R}(X)$ ) denote the right (or left) annihilator of $X$ over $R$. Also, for a ring $R$, nil $(R)$ denotes the set of all nilpotent elements of $R$ and $\operatorname{Id}(R)$ denotes the set of all idempotent elements of $R$. Furthermore, we use $\mathrm{J}(R)$ for the Jacobson radicals of a ring $R$. Recall that: A ring $R$ is called an NI-ring if nil $(R)$ is a two sided ideal in $R$ and $R$ is called a reduced ring if nil $(R)=(0)$.

According to Nicholson and Watters, in ([15], 1988), a right $R$-module $M_{R}$ is called a right $P S$-module if every simple submodule is projective, equivalently if its right socle,

$$
\operatorname{Soc}\left(M_{R}\right)=\sum\left\{B: B \text { is a simple submodule of } M_{R}\right\} .
$$

is projective. The class of PS-modules is closed under direct sums and submodules. A left PS-module ${ }_{R} M$ is defined similarly. The study of PS-modules was initiated by Gordon, in ([6], 1969). A ring $R$ is said to be a right (left) $P S$-ring if $R_{R}\left({ }_{R} R\right)$ is a right (left) PS-module. The notion of PS-rings is not left-right symmetric (see Example 11).

${ }^{*}$ Corresponding author.

Email addresses: m_farahat79@yahoo.com (Mohamed A. Farahat), SalhaAlbogamy@hotmail.com (S. T. Al-Bogamy) 
Example 1 ([15]). (a) If $\operatorname{Soc}\left(M_{R}\right)=0$, then $M_{R}$ is a right PS-module.

(b) Any projective semisimple module is a PS-module.

(c) Every regular module is a PS-module.

(d) Every nonsingular right $R$-module is a PS-module.

For any subset $X$ of $R$, the left annihilator of $X$ in a right $R$-module $M_{R}$ is denoted by

$$
\ell_{M}(X)=\{m \in M \mid m X=0\} .
$$

Similarly, one can consider the right annihilator of $X$ in a left $R$-module ${ }_{R} M$, where $X$ is any subset of $R$.

The following result is due to Weimin in ([22], 1992), which gives an equivalent condition for a right $R$-module $M_{R}$ to be a right PS-module.

Theorem 1 ([22]). The following statements are equivalent for a right $R$-module $M_{R}$ :

(1) $M_{R}\left({ }_{R} M\right)$ is a right (left) PS-module.

(2) If $L$ is a maximal right (left) ideal of $R$, then $\ell_{M}(L)=R e\left(\mathrm{r}_{M}(L)=e R\right)$, where $e \in \operatorname{Id}(R)$.

(3) If $L$ is a maximal right (left) ideal of $R$, then either $L=R e(L=e R)$, or $\ell_{M}(L)=(0)$ $\left(\mathrm{r}_{M}(L)=(0)\right)$, where $e \in \operatorname{Id}(R)$.

Example 2 ([15]). (a) Every right (left) PP-ring is a right (left) PS-ring. In particular, every right (left) Baer ring is a right (left) PS-ring.

(b) Every semiprime ring is a right (left) PS-ring. But the converse is not true.

(c) If $\ell_{R}(\mathrm{~J}(R))=0$, then $R$ is a $P S$-ring, since we have $\mathrm{J}(R) \subseteq L$ for every maximal right (left) ideal $L$ in $R$ and hence $\ell_{R}(L) \subseteq \ell_{R}(\mathrm{~J}(R))=0$ implies that for every maximal right (left) ideal $L$ in $R$, hence $\ell_{R}(L)=0$.

In [15], the class of PS-rings is closed under the formulation of polynomials and power series extensions, by other words, if a ring $R$ is a right PS-ring, then so is $R[x]$ and $R[[x]]$. The converse of this result is false by the following example:

Example 3 ([15], Example 3.2). If $R=\mathbb{Z}_{4}$, then $R[x]$ and $R[[x]]$ are PS-rings, but $R$ is not a PS-ring.

Nicholson and Watters, in [15], proved that: A ring $R$ is a right PS-ring if and only if the full matrix ring $M_{n}(R)$, where $n$ is a positive integer, is a right PS-ring. The authors in $([14], 1998)$, showed that the commutative PS-ring condition is preserved by the generalized power series rings under certain conditions. Recently, Salem, Farahat and AbdELmalk, in ([20], 2015), investigated PS-modules over Ore extensions and skew generalized power series extensions. Also, Farahat and Al-Harthy, in ([3], 2017), investigated PSmodules over generalized Mal'cev-Neumann series rings. In ([18], 2017), Paykan proved that, under suitable conditions, if $R$ is a right PS-ring, then so the skew inverse power series rings.

As a generalization of the annihilator concept, Ouyang, in ([16], 2009), introduced the weak annihilator (or nilpotent annihilator), for a nonempty subset $X$ of a ring $R$, the weak 
annihilator of $X$ in $R$ is defined as follows:

$$
\mathrm{N}_{R}(X)=\{a \in R \mid x a \in \operatorname{nil}(R) \text { for all } x \in X\} .
$$

It can be easily shown that

$$
a b \in \operatorname{nil}(R) \Leftrightarrow b a \in \operatorname{nil}(R) \text { for all } a, b \in R .
$$

Therefore there is no way to distinguish between the right weak annihilator and the left weak annihilator.

Obviously, $\mathrm{r}_{R}(X) \subseteq \mathrm{N}_{R}(X)$ for any subset $X$ of $R$ and if $R$ is reduced, then $\mathrm{r}_{R}(X)=$ $\mathrm{N}_{R}(X)$.

For example, let $D$ be an integral domain and

$$
R=T_{2}(D)=\left\{\left(\begin{array}{cc}
a & b \\
0 & c
\end{array}\right) \mid a, b, c \in D\right\}=\left(\begin{array}{cc}
D & D \\
0 & D
\end{array}\right) .
$$

We can check that nil $(R)=\left\{\left(\begin{array}{ll}0 & b \\ 0 & 0\end{array}\right) \mid b \in D\right\}=\left(\begin{array}{cc}0 & D \\ 0 & 0\end{array}\right)$ is a two sided ideal in $R$, hence $R$ is an NI-ring. Consider the subset $X=\left\{\left(\begin{array}{cc}x & 0 \\ 0 & x\end{array}\right) \mid x \in D\right\}$ of $R$. Then $\mathrm{r}_{R}(X)=0$ but $\mathrm{N}_{R}(X)=\left\{\left(\begin{array}{ll}0 & y \\ 0 & 0\end{array}\right) \mid y \in D\right\}=\left(\begin{array}{cc}0 & D \\ 0 & 0\end{array}\right)=\operatorname{nil}(R)$. Thus $\mathrm{r}_{R}(X) \varsubsetneqq$ $\mathrm{N}_{R}(X)$. Hence the weak annihilator is a nontrivial generalization of the annihilator.

Proposition 1 ([16]). Let $R$ be a ring. Then:

1) If $X \subseteq Y \subset R$, then $\mathrm{N}_{R}(Y) \subseteq \mathrm{N}_{R}(X)$,

2) $X \subseteq \mathrm{N}_{R}\left(\mathrm{~N}_{R}(X)\right)$,

3) $\mathrm{N}_{R}\left(\mathrm{~N}_{R}\left(\mathrm{~N}_{R}(X)\right)\right)=\mathrm{N}_{R}(X)$,

4) If $S \leq R$ and $X \subseteq S$, then $\mathrm{N}_{S}(X)=\mathrm{N}_{R}(X) \cap S$.

Lemma 1. Let $R$ be an NI-ring. If $a b \in \operatorname{nil}(R)$, then arb $\in \operatorname{nil}(R)$ for all $a, b, r \in R$.

Proof. Let $a, b \in R$ such that $a b \in \operatorname{nil}(R)$. Hence $b a \in \operatorname{nil}(R)$. We thus get bar $\in$ $\operatorname{nil}(R)$ for each $r \in R$. It follows that $\operatorname{arb} \in \operatorname{nil}(R)$ for each $r \in R$.

Remark 1. If $R$ is an NI-ring, then $\mathrm{N}_{R}(X)$ is an ideal of $R$ for any subset $X$ of $R$. In particular, $\mathrm{N}_{R}(a)$ is an ideal of $R$ for any $a \in R$.

In this paper, we introduce the class of weak PS-rings and investigate some of its characterization and study the transfer of weak PS-condition between a base ring $R$ and the extension ring of skew Hurwitz series $A=(H R, \sigma)$. 


\section{Rings Satisfy The Weak PS-Condition}

Motivated by the definition of the PS-condition and the definition of the weak annihilator, we introduced the notion of weak PS-condition as follows:

Definition 1. A ring $R$ satisfies the right weak $P S$-condition if, for every maximal right ideal $L$ of $R$, either $\mathrm{N}_{R}(L) \subseteq \operatorname{nil}(R)$ or $\mathrm{N}_{R}(L)=R e$, (principal left ideal generated by e), where $e \in \operatorname{Id}(R)$. Similarly, we can define the left weak PS-condition. A ring $R$ satisfies the weak PS-condition if it satisfies both the right and the left weak PS-conditions.

Remark 2. 1) In the definition of the weak PS-condition, we use "or" to mean that one of the two options only holds, but not both, unless that $\mathrm{N}_{R}(L)=(0)$. Since $\operatorname{nil}(R) \bigcap \operatorname{Id}(R)=$ (0).

2) It is clear that in reduced rings weak PS-condition and PS-condition are equivalent.

Example 4. Every domain (which is not a field) is a reduced weak PS-ring, since the left (right) annihilator of every right (left) maximal ideal is zero and hence generated by idempotent. Also, every field is a reduced weak PS-ring, since the zero ideal is the only maximal ideal in a field $F$, clearly the left (right) annihilator of the zero ideal is $F$ which is generated by 1 which is an idempotent in $F$.

Example 5. If $R$ is a commutative local ring with a unique maximal ideal $L$, then:

1) $R$ is an NI-ring and $L=\operatorname{nil}(R)$,

2) $\mathrm{N}_{R}(L)=R=\langle 1\rangle$, the principal ideal generated by 1 , which proves that a commutative local ring is a weak $P S$-ring.

3) By above we get many examples of weak PS-rings, e.g., $R=\mathbb{Z}_{p^{n}}$, for any prime number $p$ and for any positive integer $n$. The unique maximal ideal of $R=\mathbb{Z}_{p^{n}}$ is $L=\langle\bar{p}\rangle=\mathrm{J}(R)$. Also, a local domain $R=F[[x]]$, where $F$ is any field with unique maximal ideal $L=\langle x\rangle=$ $\mathrm{J}(R)$.

Example 6. Consider the commutative reduced ring $R=\mathbb{Z}_{6}$, since $\operatorname{nil}(R)=\{\overline{0}\}$. $R$ has two maximal ideals which are $L_{1}=\{\overline{0}, \overline{2}, \overline{4}\}$ and $L_{2}=\{\overline{0}, \overline{3}\}$. Note that $\operatorname{Id}(R)=\{\overline{0}, \overline{1}, \overline{3}, \overline{4}\}$. Now we compute the weak annihilator for these ideals as follows:

$$
\mathrm{N}_{R}\left(L_{1}\right)=\ell_{R}\left(L_{1}\right)=\mathrm{r}_{R}\left(L_{1}\right)=L_{2}=\{\overline{0}, \overline{3}\}=\langle\overline{3}\rangle,
$$

and

$$
\mathrm{N}_{R}\left(L_{2}\right)=\ell_{R}\left(L_{2}\right)=\mathrm{r}_{R}\left(L_{2}\right)=L_{1}=\{\overline{0}, \overline{2}, \overline{4}\}=\langle\overline{4}\rangle .
$$

Hence $\mathbb{Z}_{6}$ is a (weak) PS-ring.

Example 7. Consider the commutative reduced ring $R=\mathbb{Z}_{10}$ with $\operatorname{nil}(R)=\{\overline{0}\}$ and $\operatorname{Id}(R)=\{\overline{0}, \overline{5}, \overline{6}\}$. We can check that, $R$ has two maximal ideals only which are $L_{1}=$ $\{\overline{0}, \overline{2}, \overline{4}, \overline{6}, \overline{8}\}$ and $L_{2}=\{\overline{0}, \overline{5}\}$. Now we compute the weak annihilator for these two ideals as follows:

$$
\mathrm{N}_{R}\left(L_{1}\right)=L_{2}=\{\overline{0}, \overline{5}\}=\langle\overline{5}\rangle,
$$


and

$$
\mathrm{N}_{R}\left(L_{2}\right)=L_{1}=\{\overline{0}, \overline{2}, \overline{4}, \overline{6}, \overline{8}\}=\langle\overline{6}\rangle .
$$

Hence $\mathbb{Z}_{10}$ is a (weak) PS-ring.

Example 8. Consider the commutative nonreduced ring $R=\mathbb{Z}_{12}$ with $\operatorname{nil}(R)=\{\overline{0}, \overline{6}\}$ and $\operatorname{Id}(R)=\{\overline{0}, \overline{1}, \overline{9}\}$. We can check that, $R$ has two maximal ideals only which are $L_{1}=$ $\{\overline{0}, \overline{2}, \overline{4}, \overline{6}, \overline{8}, \overline{10}\}$ and $L_{2}=\{\overline{0}, \overline{3}, \overline{6}, \overline{9}\}$. Now we compute the weak annihilator for these two ideals as follows:

$$
\mathrm{N}_{R}\left(L_{1}\right)=L_{2}=\{\overline{0}, \overline{3}, \overline{6}, \overline{9}\}=\langle\overline{9}\rangle,
$$

and

$$
\mathrm{N}_{R}\left(L_{2}\right)=L_{1}=\{\overline{0}, \overline{2}, \overline{4}, \overline{6}, \overline{8}, \overline{10}\}=\langle\overline{2}\rangle=\langle\overline{10}\rangle .
$$

Clearly, $\mathrm{N}_{R}\left(L_{2}\right) \nsubseteq \operatorname{nil}(R)$ and $\mathrm{N}_{R}\left(L_{2}\right)$ not generated by an idempotent in $R$. Hence $\mathbb{Z}_{12}$ is not a weak PS-ring.

Now we turn to compute the left (right) annihilator for these maximal two-sided ideals as follows: $\ell_{R}\left(L_{1}\right)=\mathrm{r}_{R}\left(L_{1}\right)=\{\overline{0}, \overline{6}\}=\operatorname{nil}(R)$. Which is not generated by an idempotent element in $R$, and

$$
\ell_{R}\left(L_{2}\right)=\mathrm{r}_{R}\left(L_{2}\right)=\langle\overline{0}\rangle \text {. }
$$

Hence $\mathbb{Z}_{12}$ is not a PS-ring.

Example 9. Let $F$ be a field and $R=\left(\begin{array}{cc}F & F \\ F & F\end{array}\right)$. The only maximal right ideals (which are not left ideals) of $R$ are:

$$
L_{1}=\left(\begin{array}{cc}
F & F \\
0 & 0
\end{array}\right) \& L_{2}=\left(\begin{array}{cc}
0 & 0 \\
F & F
\end{array}\right),
$$

also,

$$
J_{1}=\left(\begin{array}{cc}
F & 0 \\
F & 0
\end{array}\right) \& 3 J_{2}=\left(\begin{array}{cc}
0 & F \\
0 & F
\end{array}\right)
$$

are the only maximal left ideals (which are not right ideals) of $R$.

$$
\operatorname{nil}(R)=\left(\begin{array}{cc}
0 & F \\
0 & 0
\end{array}\right) \bigcup\left(\begin{array}{ll}
0 & 0 \\
F & 0
\end{array}\right)
$$

which is neither a left nor a right ideal of $R$. Hence $R$ is not an NI-ring.

We can check the following facts:

1) $\mathrm{N}_{R}\left(L_{1}\right)=J_{2}=$ Re where $e=\left(\begin{array}{ll}0 & 1 \\ 0 & 1\end{array}\right) \in \operatorname{Id}(R)$,

2) $\mathrm{N}_{R}\left(L_{2}\right)=J_{1}=R f$ where $f=\left(\begin{array}{ll}1 & 0 \\ 1 & 0\end{array}\right) \in \operatorname{Id}(R)$.

Using the above discussion, we conclude that $R$ is a weak right PS-ring.

By the same way we can check the following: 
3) $\mathrm{N}_{R}\left(J_{1}\right)=L_{2}=h R$ where $h=\left(\begin{array}{ll}0 & 0 \\ 1 & 1\end{array}\right) \in \operatorname{Id}(R)$,

4) $\mathrm{N}_{R}\left(J_{2}\right)=L_{1}=k R$ where $k=\left(\begin{array}{ll}1 & 1 \\ 0 & 0\end{array}\right) \in \operatorname{Id}(R)$.

By the above discussion, we conclude that $R$ is a weak left PS-ring. Therefore $R$ is a weak PS-ring.

Example 10. Let $F$ be a filed and

$$
R=T_{2}(F)=\left(\begin{array}{cc}
F & F \\
0 & F
\end{array}\right)=\left\{\left(\begin{array}{ll}
a & b \\
0 & c
\end{array}\right) \mid a, b, c \in F\right\} .
$$

We can check that nil $(R)=\left\{\left(\begin{array}{ll}0 & b \\ 0 & 0\end{array}\right) \mid b \in F\right\}=\left(\begin{array}{cc}0 & F \\ 0 & 0\end{array}\right)$ is a two sided ideal in $R$, hence $R$ is an NI-ring. The only maximal (two sided) ideals of $R$ are:

$$
L=\left(\begin{array}{cc}
F & F \\
0 & 0
\end{array}\right) \text { and } J=\left(\begin{array}{cc}
0 & F \\
0 & F
\end{array}\right)
$$

We can check that $\mathrm{N}_{R}(L)=\operatorname{nil}(R)$ and $\mathrm{N}_{R}(J)=\operatorname{nil}(R)$. Therefore we conclude that $R$ is a weak PS-ring.

Example 11. Consider the semigroup ring

$$
V=\mathbb{Z}_{2}[S]=\left\{\alpha a+\beta b: \alpha, \beta \in \mathbb{Z}_{2}\right\}=\{0, a, b, a+b=c\},
$$

where $S=\{a, b\}$ is the semigroup with the following Cayley multiplication table

$$
\begin{array}{ccc}
\times & a & b \\
a & a & b \\
b & a & b
\end{array}
$$

Clearly,

$$
\operatorname{Id}(V)=\{0, a, b\} \mathscr{E} \operatorname{nil}(V)=\{0, c\} .
$$

We can check the following:

1) $I=\{0, a\}$ is a left maximal ideal in $V$ which is not a right ideal,

2) $J=\{0, b\}$ is a left maximal ideal in $V$ which is not a right ideal,

3) $K=\{0, c\}=\operatorname{nil}(V)$ is a maximal 2-sided ideal in $V$, hence $V$ is an NI-ring,

4) We can check that $\mathrm{N}_{V}(I)=K=\operatorname{nil}(V), \mathrm{N}_{V}(J)=K=\operatorname{nil}(V)$ and $\mathrm{N}_{V}(K)=V=$ $a \times V=b \times V$. Therefore $V$ is a weak left PS-ring. Since the only right maximal ideal in $V$, is $K=\{0, c\}=\operatorname{nil}(V)$ and $\mathrm{N}_{V}(K)=V$ which is not generated by an idempotent as a left ideal, so $V$ is not a right weak PS-ring.

Also, we can check the following: $\mathrm{r}_{V}(I)=0=0 \times V, \mathrm{r}_{V}(J)=0=0 \times V$, and $\mathrm{r}_{V}(K)=V=a \times V=b \times V$. Hence $V$ is a left PS-ring. For the unique maximal right ideal $K$ in $V$, we have $\ell_{V}(K)=V$ which is not generated by an idempotent as a left ideal. Hence $V$ is not a right PS-ring. 
Lemma 2. Let $R$ be an NI-ring, $L$ a maximal right ideal in $R$ and $\bar{R}=R / \operatorname{nil}(R)$. Then:

1) $L$ contains $N=\operatorname{nil}(R)$,

2) $\bar{L}=L / N$ is a maximal right ideal in $\bar{R}$, and

3) If $e \in \operatorname{Id}(R)$, then $\bar{e} \in \operatorname{Id}(\bar{R})$.

Proof. 1) Let the contrary, i.e., $N=\operatorname{nil}(R) \nsubseteq L$, then there is $0 \neq a \in N$, such that $a \notin L$. So $L+a R=R$, thus

$$
b+a r=1, \text { for some } b \in L \text { and } r \in R .
$$

Since every nilpotent element lies in $\mathrm{J}(R)$, we have $b=1-a r$ is a right invertible element in $R$, which impossible since $b \in L$ and $L$ is a maximal right ideal in $R$.

2) Let the contrary, i.e., $\bar{L}=L / N$ is not a maximal right ideal in $\bar{R}$, then there is a proper right ideal $\bar{K}=K / N$ in $\bar{R}$, for some right ideal $K$ in $R$ contain $N$, such that $\bar{L} \subset \bar{K}$ in $\bar{R}$, which implies clearly that $L \subset K$ in $R$, which contradicts for the maximality of $L$.

3) Let $e \in \operatorname{Id}(R)$, then $(\bar{e})^{2}=(e+N)(e+N)=e^{2}+N=e+N=\bar{e}$, hence $\bar{e} \in \operatorname{Id}(\bar{R})$.

Theorem 2. Suppose that $R$ is an NI-ring. If $R$ is a weak right PS-ring, then $\bar{R}=$ $R /$ nil $(R)$ is a right PS-ring.

Proof. Let $R$ be a weak right PS-ring and $L$ be a maximal right ideal in $R$. From Lemma 2, we have $L$ contains $N$ and $\bar{L}=L / N$ is a maximal right ideal in $\bar{R}$. Since $R$ is a weak right PS-ring, we have either $\mathrm{N}_{R}(L) \subseteq \operatorname{nil}(R)$ or $\mathrm{N}_{R}(L)=R e$, for some $e \in \operatorname{Id}(R)$. Case (1): Assume first that $\mathrm{N}_{R}(L) \subseteq \operatorname{nil}(R)$. Let $\bar{a} \in \ell_{\bar{R}}(\bar{L})$. Then $\bar{a} \bar{L}=\overline{0}=\operatorname{nil}(R)$, which implies that $a L \subseteq \operatorname{nil}(R)$. Hence $a \in \mathrm{N}_{R}(L) \subseteq \operatorname{nil}(R)$. Thus $\bar{a}=\overline{0}$ which implies that $\ell_{\bar{R}}(\bar{L})=(\overline{0})$ and we conclude that $\bar{R}=R / \operatorname{nil}(R)$ is a right PS-ring.

Case (2): Assume that $\mathrm{N}_{R}(L)=R e$, for some $e \in \operatorname{Id}(R)$. Let $\bar{a} \in \ell_{\bar{R}}(\bar{L})$. Then $\bar{a} \bar{L}=\overline{0}=\operatorname{nil}(R)$, which implies that $a L \subseteq \operatorname{nil}(R)$. Hence $a \in \mathrm{N}_{R}(L)=R e$ and so $a=r e$, for some $r \in R$. Therefore $\bar{a}=\overline{r e}$. Thus $\ell_{\bar{R}}(\bar{L})=\bar{R} \bar{e}$ and we conclude that $\bar{R}=R / \operatorname{nil}(R)$ is a right PS-ring.

Example 12. In Example 8 we conclude that the ring $R=\mathbb{Z}_{12}$ is not a weak PS-ring. We can check that $\bar{R}=R / \operatorname{nil}(R) \cong \mathbb{Z}_{6}$, which, by Example 6 , is a weak PS-ring. So we get here an example to show that the converse direction of Theorem 2 does not hold.

Example 13. In Example 11 we conclude that the ring $R=V$ is a weak left $P S$-ring. We turn now to check the condition of weak PS-ring on the direct sum $A=V \oplus V$. In $A$, we have:

$$
\begin{aligned}
\operatorname{nil}(A) & =\operatorname{nil}(V) \oplus \operatorname{nil}(V)=\{(0,0),(0, c),(c, 0),(c, c)\}, \text { and } \\
\operatorname{Id}(A) & =\operatorname{Id}(V) \oplus \operatorname{Id}(V) \\
& =\{(0,0),(0, a),(a, 0),(0, b),(b, 0),(a, b),(b, a)\} .
\end{aligned}
$$

Consider now the following maximal two sided ideal in A,

$$
L=K \oplus V=\{(0,0),(0, c),(a, 0),(a, c),(b, 0),(b, c),(c, 0),(c, c)\} .
$$


By direct computations we get

$$
\begin{aligned}
\mathrm{N}_{A}(K \oplus V) & =\mathrm{N}_{V}(K) \oplus \mathrm{N}_{V}(V)=V \oplus K \\
& =\{(0,0),(0, a),(0, b),(0, c),(c, 0),(c, a),(c, b),(c, c)\}
\end{aligned}
$$

Clearly, $\mathrm{N}_{A}(K \oplus V)$ is not contained in nil $(A)$ and not generated by idempotent in $A$. Therefore $A=V \oplus V$ is not a weak left (right) PS-ring, i.e., the direct sum of weak left (right) PS-rings not necessary be a weak left (right) PS-ring.

Recall that a nonzero right ideal $I$ of $R$ is a right essential ideal if $I$ has nonzero intersection with every nonzero right ideal of $R$. A singular right ideal $\mathrm{S}_{\mathrm{r}}(R)$ of $R$ is defined by

$$
\mathrm{S}_{\mathrm{r}}(R)=\left\{a \in R \mid \mathrm{r}_{R}(a) \text { is an essential right ideal of } R\right\} .
$$

Similarly, we can define a singular left ideal $\mathrm{S}_{\ell}(R)$ of $R$ is defined by

$$
\mathrm{S}_{\ell}(R)=\left\{a \in R \mid \ell_{R}(a) \text { is an essential left ideal of } R\right\} .
$$

A ring $R$ is called right (left) nonsingular if $\mathrm{S}_{\mathrm{r}}(R)=0\left(\mathrm{~S}_{\ell}(R)=0\right)$ and right (left) singular if $\mathrm{S}_{\mathrm{r}}(R)=R\left(\mathrm{~S}_{\ell}(R)=R\right)$.

We define a weak singular ideal of an NI-ring $R$ as follows:

$$
\mathrm{NS}(R)=\left\{a \in R \mid \mathrm{N}_{R}(a) \text { is an essential ideal of } R\right\} .
$$

We extend the definitions of a right (left) nonsingular ring and a right (left) singular ring to a weak nonsingular ring and a weak singular ring, respectively, as follows:

Definition 2. An NI-ring $R$ is called a weak singular ring if $\mathrm{NS}(R)=R$ and $R$ is called a weak nonsingular ring if $\mathrm{NS}(R)=0$.

Example 14. 1) In example 5, $R=\mathbb{Z}_{4}$, we can check that $\mathrm{NS}(R)=R$, hence $R$ is a weak singular ring. But $\mathrm{S}_{\mathrm{r}}(R)=\mathrm{S}_{\ell}(R)=\{\overline{0}, \overline{2}\}=\operatorname{nil}(R)$, hence $R$ is neither a nonsingular ring nor a singular ring.

2) In example $6, R=\mathbb{Z}_{6}$, we can check that $\mathrm{NS}(R)=\mathrm{S}_{\mathrm{r}}(R)=\mathrm{S}_{\ell}(R)=(0)$, hence $R$ is a (weak) nonsingular ring.

3) In example $7, R=\mathbb{Z}_{10}$, we can check that $\mathrm{NS}(R)=\mathrm{S}_{\mathrm{r}}(R)=\mathrm{S}_{\ell}(R)=(0)$, hence $R$ is a (weak) nonsingular ring.

4) In example $8, R=\mathbb{Z}_{12}$, we can check that $\mathrm{NS}(R)=\{\overline{0}, \overline{6}\}=\operatorname{nil}(R)$, hence $R$ is neither a weak nonsingular ring nor a weak singular ring. But $\mathrm{S}_{\mathrm{r}}(R)=\mathrm{S}_{\ell}(R)=\{\overline{0}, \overline{6}\}=\operatorname{nil}(R)$, hence $R$ is neither a nonsingular ring nor a singular ring.

5) In example 11, $R=V$, we can check that $\mathrm{NS}(V)=\{0, c\}=K=\operatorname{nil}(V)$, hence $V$ is neither a weak nonsingular ring nor a weak singular ring. But $\mathrm{S}_{\mathrm{r}}(V)=\{0, c\}=K$ and $\mathrm{S}_{\ell}(V)=(0)$, hence $V$ is a left nonsingular ring which is not a right nonsingular ring.

Lemma 3. For any ring $R$, we have $\mathrm{S}_{\mathrm{r}}(R) \subseteq \mathrm{NS}(R)$. 
Proof. If $a \in \mathrm{S}_{\mathrm{r}}(R)$, then $\mathrm{r}_{R}(a)$ is an essential right ideal. Since $\mathrm{r}_{R}(a) \subseteq \mathrm{N}_{R}(a)$, we conclude that $\mathrm{N}_{R}(a)$ is also essential ideal. Thus $a \in \mathrm{NS}(R)$. Therefore $\mathrm{S}_{\mathrm{r}}(R) \subseteq \mathrm{NS}(R)$.

Corollary 1. 1) Every weak nonsingular ring is a right (left) nonsingular ring.

2) Every right (left) singular ring is a weak singular ring.

Remark 3. The converse of the last Corollary need not be true by the following examples: 1) In example $14,(1), R=\mathbb{Z}_{4}$, is a weak singular ring. But $R$ is not a singular ring.

2) In example 14, (5), $R=V$, is a left nonsingular ring. But $R$ is not a weak singular ring.

Corollary 2. Every weak nonsingular ring is a PS-ring.

Proof. Since every nonsingular ring is a PS-ring, the result follows.

Theorem 3. A semisimple NI-ring is a weak right (left) PS-ring.

Proof. It is well-known that any right (resp. left) ideal $I$ in a semisimple ring $R$ has the form $I=e R$ (resp. $I=R e$ ), where $e \in \operatorname{Id}(R)$. Since $R$ is an NI-ring, we have $\mathrm{N}_{R}(L)$ is an ideal of $R$ and the proof is complete.

Remark 4. A ring $R$, in Example 9, is a simple ring, hence $R$ is a semisimple ring which is not an NI-ring. But $R$ is a weak right (left) PS-ring.

Example 15. In Example 11, $R=V$, we have $V$ is a left nonsingular ring which is not a right nonsingular ring. Therefore $V$ is a left PS-ring which is not a reduced ring.

Given a ring $R$ and ${ }_{R} M_{R}$ an $R$ - $R$-bimodule, the trivial extension of $R$ by $M$ is the ring $T(R, M)=R \oplus M$ with the usual addition and the multiplication

$$
\left(r_{1}, m_{1}\right)\left(r_{2}, m_{2}\right)=\left(r_{1} r_{2}, r_{1} m_{2}+m_{1} r_{2}\right), \text { where } r_{1}, r_{2} \in R \text { and } m_{1}, m_{2} \in M .
$$

This is isomorphic to the ring of all matrices $\left(\begin{array}{cc}r & m \\ 0 & r\end{array}\right)$, where $r \in R$ and $m \in M$ with the usual matrix operations.

Trivial extensions attracted attention when people searched for nonreduced rings which are Armendraiz [2]. The article of Rege and Chhawchharia ([19], 1997) seems to be the first to consider the Armendraiz property of trivial extensions.

Proposition 2. Let $R$ be a reduced ring. If $T(R, M)$ is a weak right PS-ring, then $R$ is a right PS-ring.

Proof. Set $A=T(R, M)$. Since $R$ is a reduced ring, we can easily conclude that $\operatorname{nil}(A) \cong\left\{\left(\begin{array}{cc}0 & m \\ 0 & 0\end{array}\right) \mid m \in M\right\}$ and hence $\bar{A}=A /$ nil $(A) \cong R$, which completes the proof. 
Proposition 3. Let $R$ be a reduced ring. If $T(R, R)$ is a weak right PS-ring, then $R$ is a right PS-ring.

Observe that $T(R, R) \cong R[x] /\left\langle x^{2}\right\rangle$, so we get the following Corollary:

Corollary 3. Let $R$ be a reduced ring. If $R[x] /\left\langle x^{2}\right\rangle$ is a weak right PS-ring, then $R$ is a right PS-ring.

\section{Skew Hurwitz Series Rings Satisfy The Weak PS-Condition}

Rings of formal power series have been of interest and have had important applications in many areas, one of which has been differential algebra. In an earlier paper by Keigher [10], the ring of Hurwitz series, a variant of the ring of formal power series was considered, and some of its properties, especially its categorical properties, were studied. In the papers $([11,12])$ Keigher demonstrated that the ring of Hurwitz series has many interesting applications in differential algebra and in the discussion about weak normalization. The product of series using the binomial coefficients, was studied also in papers by Fliess [5] and Taft [21]. Ring-theoretical properties of Hurwitz series rings and its skew have been investigated by many authors $([10,11,12,7,8,4])$. In this section we study the skew Hurwitz series rings over a noncommutative rings and examine their structures and properties.

The elements of $H R$ are ordinary formal series, i.e.,

$$
H R=\left\{\sum_{i=0}^{\infty} a_{i} x^{i} \mid a_{i} \in R\right\} .
$$

The operation of addition in $H R$ is a componentwise addition and the operation of multiplication is defined by the following:

For each two Hurwitz series $f(x)=\sum_{i=0}^{\infty} a_{i} x^{i}$ and $g(x)=\sum_{j=0}^{\infty} b_{j} x^{j} \in H R$,

$$
\left(\sum_{i=0}^{\infty} a_{i} x^{i}\right)\left(\sum_{j=0}^{\infty} b_{j} x^{j}\right)=\sum_{n=0}^{\infty}\left(\sum_{i=0}^{n}\left(\begin{array}{l}
n \\
i
\end{array}\right) a_{i} b_{n-i}\right) x^{n},
$$

where $\left(\begin{array}{c}n \\ i\end{array}\right)=C_{i}^{n}=\frac{n !}{i ! \times(n-i) !}$, is the binomial coefficient. From the above discussion we can say that: The multiplication is subject to the relation (Hurwitz multiplication rule)

$$
\left(a_{i} x^{i}\right)\left(b_{j} x^{j}\right)=\left(\begin{array}{c}
i+j \\
i
\end{array}\right) a_{i} b_{j} x^{i+j}=\left(\begin{array}{c}
i+j \\
j
\end{array}\right) a_{i} b_{j} x^{i+j}
$$

It is now routine to check that $H R$ is a ring with identity $1_{R}$ which is called the Hurwitz series rings over $R$. Clearly, $R$ is a subring of $H R$.

Despite the apparent similarity between the formal power series rings $R[[x]]$ and the Hurwitz series rings $H R$ but there are substantial difference between them for example, 
the ideal $\left\langle x, x^{2}, x^{3}, \ldots\right\rangle$ is principal in $R[[x]]$ which is the same as the ideal $\langle x\rangle$ but the ideal $\left\langle x, x^{2}, x^{3}, \ldots\right\rangle$ in $H R$ is not a finitely generated. Here $x^{2} \notin\langle x\rangle$ since $x . x=2 x^{2}$. Clearly, if $R$ has $\mathbb{Q}$ as a subring, then the ideal $\left\langle x, x^{2}, x^{3}, \ldots\right\rangle$ in $H R$ will be the same as the principal ideal $\langle x\rangle$.

Let $\sigma$ be an endomorphism of the ring $R$, with $\sigma(1)=1$. The elements of $A=(H R, \sigma)$, the ring of skew Hurwitz series, are the ordinary formal series $\sum_{i=0}^{\infty} a_{i} x^{i}$, where $a_{i} \in R$, with component wise addition and the following operation of multiplication:

For each two Hurwitz series $f(x)=\sum_{i=0}^{\infty} a_{i} x^{i}$ and $g(x)=\sum_{j=0}^{\infty} b_{j} x^{j} \in A=(H R, \sigma)$,

$$
\left(\sum_{i=0}^{\infty} a_{i} x^{i}\right)\left(\sum_{j=0}^{\infty} b_{j} x^{j}\right)=\sum_{n=0}^{\infty}\left(\sum_{i=0}^{n}\left(\begin{array}{c}
n \\
i
\end{array}\right) a_{i} \sigma^{i}\left(b_{n-i}\right)\right) x^{n} .
$$

From the above discussion we can say that: The multiplication is subject to the relation (Hurwitz multiplication rule)

$$
\left(a_{i} x^{i}\right)\left(b_{j} x^{j}\right)=\left(\begin{array}{c}
i+j \\
i
\end{array}\right) a_{i} \sigma^{i}\left(b_{j}\right) x^{i+j}=\left(\begin{array}{c}
i+j \\
j
\end{array}\right) a_{i} \sigma^{i}\left(b_{j}\right) x^{i+j}
$$

By $h R$ and $P=(h R, \sigma)$, we denote the polynomial Hurwitz ring and skew polynomial Hurwitz ring, respectively. The product, in this case will be as follows: For each two polynomial Hurwitz $f(x)=\sum_{i=0}^{v} a_{i} x^{i}$ and $g(x)=\sum_{j=0}^{u} b_{j} x^{j} \in P$,

$$
\left(\sum_{i=0}^{v} a_{i} x^{i}\right)\left(\sum_{j=0}^{u} b_{j} x^{j}\right)=\sum_{n=0}^{v+u}\left(\sum_{i=0}^{n}\left(\begin{array}{c}
n \\
i
\end{array}\right) a_{i} \sigma^{i}\left(b_{n-i}\right)\right) x^{n}
$$

Annin [1] introduced the notion of $\sigma$-compatibility of rings as follows.

Definition $3([1])$. A ring $R$ is said to be $\sigma$-compatible if $a b=0 \Leftrightarrow a \sigma(b)=0$, where $a, b \in R$.

Some of the basic properties of a $\sigma$-compatible ring was given in the following lemmas.

Lemma 4 ([1]). If $R$ is a $\sigma$-compatible ring, then:

1) $\sigma(1)=1$,

2) $\sigma$ is a monomorphism,

3) $a b=0 \Leftrightarrow \sigma(a) b=0$, where $a, b \in R$.

Lemma 5 ([1]). Let $R$ be a $\sigma$-compatible ring. Then $a b=0 \Leftrightarrow a \sigma^{i}(b)=0 \Leftrightarrow \sigma^{i}(a) b=0$ for all $i \geq 0$ and $a, b \in R$.

In [13], the author introduced the concept of $\sigma$-rigid rings for rings with an endomorphism $\sigma$, as follows: 
Definition 4 ([13]). Let $R$ be a ring with an endomorphism $\sigma$. If $a \sigma(a)=0 \Rightarrow a=0$, for $a \in R$, then $R$ is called a $\sigma$-rigid ring.

Some of the basic properties of a $\sigma$-rigid ring was given in the following lemma.

Lemma 6 ([9]). If $R$ is a $\sigma$-rigid ring, then:

1) $R$ is a reduced ring,

2) $\sigma$ is a monomorphism.

The relation between a $\sigma$-compatible ring and a $\sigma$-rigid ring was studied in the following lemma.

Lemma 7 ([9]). A ring $R$ is a reduced $\sigma$-compatible ring if and only if $R$ is $\sigma$-rigid.

Hong, et. al., in ([9], Proposition 5), proved that: If $R$ is a $\sigma$-rigid ring, then for each $e \in \operatorname{Id}(R)$, we have $\sigma(e)=e$.

In what follows, we characterize skew Hurwitz series rings that satisfy the weak PScondition. We shall need the following auxiliary result in the proof of our Theorems.

Lemma 8 (2.5 and $2.6[17])$. Let $R$ be a $\sigma$-compatible NI-ring with nil $(R)$ nilpotent and $R$ a torsion free as a $\mathbb{Z}$-module. Set $K=\operatorname{nil}(R)$. Then we have:

(1) $\operatorname{nil}(A)=(H K, \sigma)$.

(2) $f(x)=\sum_{i=0}^{\infty} a_{i} x^{i} \in \operatorname{nil}(A)$ if and only if $a_{i} \in \operatorname{nil}(R)$ for all $i \geq 0$.

(3) If $f(x)=\sum_{i=0}^{\infty} a_{i} x^{i}$ and $g(x)=\sum_{j=0}^{\infty} b_{j} x^{j} \in A$ such that $f(x) g(x) \in \operatorname{nil}(A)$, then $a_{i} b_{j} \in K=\operatorname{nil}(R)$ for all $i, j \geq 0$.

As a corollary we get the following result in the finite case.

Lemma 9. Let $R$ be a $\sigma$-compatible NI-ring with $\operatorname{nil}(R)$ nilpotent and $R$ a torsion free as $a \mathbb{Z}$-module. Set $K=\operatorname{nil}(R)$. Then we have:

(1) $\operatorname{nil}(P)=(h K, \sigma)$.

(2) $f(x)=\sum_{i=0}^{k} a_{i} x^{i} \in \operatorname{nil}(P)$ if and only if $a_{i} \in \operatorname{nil}(R)$ for all $i \geq 0$.

(3) If $f(x)=\sum_{i=0}^{k} a_{i} x^{i}$ and $g(x)=\sum_{j=0}^{n} b_{j} x^{j} \in P$ such that $f(x) g(x) \in \operatorname{nil}(P)$, then $a_{i} b_{j} \in K=\operatorname{nil}(R)$ for all $0 \leq i \leq k$ and $0 \leq j \leq n$.

Now, we can prove our result in the infinite case:

Theorem 4. Let $R$ be a $\sigma$-compatible NI-ring with $\operatorname{nil}(R)$ nilpotent, $\sigma(e)=e$ for every $e \in \operatorname{Id}(R)$ and $R$ a torsion free as a $\mathbb{Z}$-module. If $R$ is a weak right PS-ring, then $A=$ $(H R, \sigma)$ is a weak right PS-ring. 
Proof. Let $L$ be a maximal right ideal of $A=(H R, \sigma)$. We will show that either $\mathrm{N}_{A}(L) \subseteq \operatorname{nil}(A)$ or $\mathrm{N}_{A}(L)=A q$, where $q \in \operatorname{Id}(A)$. Let $I$ be the set of all coefficients of all Hurwitz series in $L$ and let $J$ be the right ideal of $R$ generated by $I$, i.e.,

$$
J=\langle I\rangle_{r}=I R
$$

If $J=R$, then there exist $a_{1}, a_{2}, \ldots, a_{n} \in I$ and $r_{1}, r_{2}, \ldots, r_{n} \in R$, such that

$$
1=a_{1} r_{1}+a_{2} r_{2}+\ldots+a_{n} r_{n}
$$

Suppose that $\varphi(x)=\sum_{i=0}^{\infty} b_{i} x^{i} \in \mathrm{N}_{A}(L)$, then for every $f(x)=\sum_{j=0}^{\infty} a_{j} x^{j} \in L$, we have

$$
\varphi(x) f(x)=\left(\sum_{i=0}^{\infty} b_{i} x^{i}\right)\left(\sum_{j=0}^{\infty} a_{j} x^{j}\right) \in \operatorname{nil}(A) .
$$

Since $R$ is a $\sigma$-compatible NI-ring with $\operatorname{nil}(R)$ a nilpotent and $R$ a torsion free as a $\mathbb{Z}$ module from Lemma 8, we get that $b_{i} a_{j} \in \operatorname{nil}(R)$, for all integers $0 \leq i$ and $0 \leq j$. Consequently, for every $a \in I, b_{i} a \in \operatorname{nil}(R)$, for all integers $0 \leq i$. Hence $b_{i} \in \mathrm{N}_{R}(J)=$ $\mathrm{N}_{R}(R)=\operatorname{nil}(R)$, for all integers $0 \leq i$. Therefore $\varphi(x) \in \operatorname{nil}(A)$. Hence $\mathrm{N}_{A}(L) \subseteq \operatorname{nil}(A)$. If $J \neq R$, we show that $J$ is a maximal right ideal of $R$. Let $r \in R-J$. If $r \in L$, then $r \in I$ and so $r \in J$, which is a contradiction. Thus $r \notin L$. Since $L$ is a maximal right ideal of $A$, we have

$$
A=L+r A \text {. }
$$

It follows that there exist $f(x)=\sum_{i=0}^{\infty} a_{i} x^{i} \in L$ and $g(x)=\sum_{j=0}^{\infty} b_{j} x^{j} \in A$, such that

$$
1=a_{0}+r b_{0} .
$$

If $a_{0}=0$, then $1=r b_{0} \in r . R$ and so $R=J+r R$.

If $a_{0} \neq 0$, then $a_{0} \in I \subset J$ which implies that $R=J+r R$. Hence $J$ is a maximal right ideal of $R$.

Since $R$ is a weak right PS-ring, it follows that either $\mathrm{N}_{R}(J) \subseteq \operatorname{nil}(R)$ or $\mathrm{N}_{R}(J)=R e$, where $e \in \operatorname{Id}(R)$.

Case (1): Assume that $\mathrm{N}_{R}(J) \subseteq \operatorname{nil}(R)$. We will show that $\mathrm{N}_{A}(L) \subseteq \operatorname{nil}(A)$. Let $\varphi(x)=$ $\sum_{i=0}^{\infty} m_{i} x^{i} \in \mathrm{N}_{A}(L)$. Then for every $g(x)=\sum_{j=0}^{\infty} a_{j} x^{j} \in L$, we have

$$
\varphi(x) g(x)=\left(\sum_{i=0}^{\infty} b_{i} x^{i}\right)\left(\sum_{j=0}^{\infty} a_{j} x^{j}\right) \in \operatorname{nil}(A) .
$$

Since $R$ is a $\sigma$-compatible NI-ring with $\operatorname{nil}(R)$ a nilpotent, from Lemma 8 , we get that $b_{i} a_{j} \in \operatorname{nil}(R)$, for all integers $0 \leq i$ and $0 \leq j$. Consequently, for every $a \in I, b_{i} a \in \operatorname{nil}(R)$, 
for all integers $0 \leq i$. Hence $b_{i} \in \mathrm{N}_{R}(J)=\mathrm{N}_{R}(R)=\operatorname{nil}(R)$, for all integers $0 \leq i$. Therefore $\varphi(x) \in \operatorname{nil}(A)$ and we have $\mathrm{N}_{A}(L) \subseteq \operatorname{nil}(A)$.

Case (2): Assume that $\mathrm{N}_{R}(J)=R e$, where $e \in \operatorname{Id}(R)$. We will show that $\mathrm{N}_{A}(L)=A q$, where $q \in \operatorname{Id}(A)$. Let $\varphi(x)=\sum_{i=0}^{\infty} b_{i} x^{i} \in \mathrm{N}_{A}(L)$ and $\varphi(x) \notin \operatorname{nil}(A)$, then for every $f(x)=$ $\sum_{j=0}^{\infty} a_{j} x^{j} \in L$, we have

$$
\varphi(x) f(x)=\left(\sum_{i=0}^{\infty} b_{i} x^{i}\right)\left(\sum_{j=0}^{\infty} a_{j} x^{j}\right) \in \operatorname{nil}(A) .
$$

Since $R$ is a $\sigma$-compatible NI-ring with $\operatorname{nil}(R)$ a nilpotent, from Lemma 8 , we get that $b_{i} a_{j} \in \operatorname{nil}(R)$, for all integers $0 \leq i$ and $0 \leq j$. Consequently, for every $a \in I, b_{i} a \in \operatorname{nil}(R)$, for all integers $0 \leq i$. For any $m \in J$, there exist $a_{1}, a_{2}, \ldots, a_{n} \in I$ and $r_{1}, r_{2}, \ldots, r_{n} \in R$, such that

$$
\begin{aligned}
m & =a_{1} r_{1}+a_{2} r_{2}+\ldots+a_{n} r_{n}, \\
b_{i} m & =\left(b_{i} a_{1}\right) r_{1}+\left(b_{i} a_{2}\right) r_{2}+\ldots+\left(b_{i} a_{n}\right) r_{n},
\end{aligned}
$$

hence $b_{i} m \in \operatorname{nil}(R)$, for all integers $0 \leq i$, so $b_{i} \in \mathrm{N}_{R}(J)=R e$, for all integers $0 \leq i$. Therefore there exist $t_{i} \in R$ such that $b_{i}=t_{i} e$, for all integers $0 \leq i$, and we have $\sigma(e)=e$. Hence

$$
\varphi(x)=\sum_{i=0}^{\infty} b_{i} x^{i}=\sum_{i=0}^{\infty} t_{i} e x^{i}=\left(\sum_{i=0}^{\infty} t_{i} x^{i}\right) e \in A q, \text { where } q^{2}=e^{2}=e=q \in A .
$$

Therefore $\mathrm{N}_{A}(L)=A q$, where $q \in \operatorname{Id}(A)$ and the result is proved.

As a corollary we get the following result in the finite case.

Corollary 4. Let $R$ be a $\sigma$-compatible NI-ring with $\operatorname{nil}(R)$ nilpotent, $\sigma(e)=e$ for every $e \in \operatorname{Id}(R)$ and $R$ a torsion free as a $\mathbb{Z}$-module. If $R$ is a weak right $P S$-ring, then $P=$ $(h R, \sigma)$ is a weak right $P S$-ring.

Theorem 5. Let $R$ be a $\sigma$-compatible NI-ring with $\operatorname{nil}(R)$ nilpotent and $R$ a torsion free as a $\mathbb{Z}$-module. If $R$ is a weak left PS-ring, then $A=(H R, \sigma)$ is a weak left PS-ring.

Proof. The proof is similar to the previous proof of Theorem 4. The only thing we need to note here is that, if $L$ is a maximal left ideal of $A=(H R, \sigma)$, then, by the analogue manner as above, we get in case (2) that $b_{i} \in \mathrm{N}_{R}(J)=e R$, for all integers $0 \leq i$. Therefore there exist $t_{i} \in R$ such that $b_{i}=e_{i}$, for all integers $0 \leq i$. So

$$
\varphi(x)=\sum_{i=0}^{\infty} b_{i} x^{i}=\sum_{i=0}^{\infty} e t_{i} x^{i}=e\left(\sum_{i=0}^{\infty} t_{i} x^{i}\right) \in q A, \text { where } q^{2}=e^{2}=e=q \in A .
$$

Therefore $\mathrm{N}_{A}(L)=q A$, where $q \in \operatorname{Id}(A)$ and the result is proved.

As a corollary we get the following result in the finite case. 
Corollary 5. Let $R$ be a $\sigma$-compatible NI-ring with $\operatorname{nil}(R)$ nilpotent and $R$ a torsion free as a $\mathbb{Z}$-module. If $R$ is a weak left PS-ring, then $P=(h R, \sigma)$ is a weak left PS-ring.

Assume that $\sigma$ is the identity map, then the skew Hurwitz series ring $A=(H R, \sigma)$ is $H R$, the usual Hurwitz series ring over $R$, and the skew Hurwitz polynomial $\operatorname{ring} P=$ $(h R, \sigma)$ is $h R$, the usual Hurwitz polynomial ring over $R$, so we get the following corollaries:

Corollary 6. Let $R$ be an NI-ring with $\operatorname{nil}(R)$ nilpotent and $R$ a torsion free as a $\mathbb{Z}$ module. If $R$ is a weak right (left) PS-ring, then usual Hurwitz series ring over $H R$ is a weak right (left) PS-ring.

Corollary 7. Let $R$ be an NI-ring with $\operatorname{nil}(R)$ nilpotent and $R$ a torsion free as a $\mathbb{Z}$ module. If $R$ is a weak right (left) PS-ring, then usual Hurwitz polynomial ring over $h R$ is a weak right (left) PS-ring.

\section{Acknowledgements}

The authors wish to express their sincere thanks to the referee for his/her helpful comments and valuable suggestions.

\section{References}

[1] S. Annin. Associated primes over ore extension rings. J. Algebr. Appl., 3:193-205, 2004.

[2] E. Armendariz. A note on extensions of Baer and p.p.-ring. J. Aust. Math. Soc., 18:470-473, 1974.

[3] M. Farahat and N. Al-Harthy. PS-modules of generalized Mal'cev-Neumann series rings. Hacettepe Journal of Mathematics and Statistics, 46(5):1-6, 2017.

[4] M. Farahat and S. Al-Nafaie. Some results on skew Hurwitz series rings. Far East Journal of Mathematical Sciences, 101(12):2767-2784, 2017.

[5] M. Fliess. Sur divers produits de series fonnelles. Bull. Soc. Math. Fr., 102:181-191, 1974.

[6] R. Gordon. Rings in which minimal left ideals are projective. Pac. J. Math., 31:679$692,1969$.

[7] E. Hashemi. Extensions of Baer and quasi-Baer modules. Bull. Iranian Math. Soc., $37(1): 1-13,2011$.

[8] A. Hassanein and M. Farahat. Some properties of skew Hurwitz series. Le Matematiche, LXIX(I):169-178, 2014. 
[9] C. Hong, N. Kim, and T. Kwak. Ore extensions of Baer and p.p.-rings . J. Pure and Appl. Alg., 151:215-226, 2000.

[10] W. Keigher. Adjunctions and comonads in differential algebra . Pacific. J. Math, 248:99-112, 1975.

[11] W. Keigher. On the ring of Hurwitz series . Comm. Alg., 25(6):1845-1859, 1997.

[12] W. Keigher and F. Pritchard. Hurwitz series as formal functions . J. Pure Appl. Alg., 146:291-304, 2000.

[13] J. Krempa. Some examples of reduced rings . Algebra Colloq., 3:289-300, 1996.

[14] Z. Liu and F. Li. PS-rings of generalized power series . Comm. Algebra, 26:2283-2291, 1998.

[15] W. Nicholson and J. Watters. Rings with projective socle. Proc. Amer. Math. Soc., 102:443-450, 1988.

[16] L. Ouyang. Ore extensions of weak zip rings. Glasg. Math. J., 51:525-537, 2009.

[17] K. Paykan. Nilpotent elements of skew Hurwitz series rings . Rend. Circ. Mat. Palermo., 2016.

[18] K. Paykan. Skew inverse power series rings over a ring with projective socle . Czec. Math. J., 67(142):389-398, 2017.

[19] M. Rege and S. Chhawchharia. Armendariz rings . Proc. Japan Acad. Ser. A Math. Sci., 73:14-17, 1997.

[20] R. Salem, M. Farahat, and H. Abd-ELmalk. PS-modules over Ore extensions and skew generalized power series rings. Int. J. Math. Math. Sci., 2015.

[21] E. Taft. Hurwitz invertibility of linearly recursive sequences. Congr. Numerantium, $73: 37-40,1990$.

[22] X. Weimin. Modules with projective socles. Riv. Math. Univ. Parma (N.S.), 1(5):311$315,1992$. 